\section{The NCCN 22nd Annual Conference: Discussing Treatment Disparities, the Doctor-Patient Relationship, the Latest NCCN Guidelines Updates, and More}

\author{
Robert W. Carlson, MD
}

The NCCN 22nd Annual Conference: Improving the Quality, Effectiveness, and Efficiency of Cancer Care attracted more than 1,650 registrants from across the United States and the globe, including oncologists (in both community and academic settings), oncology fellows, nurses, pharmacists, and other healthcare professionals. The breadth of topics presented at the conference is a reflection of the impact NCCN and the NCCN Clinical Practice Guidelines in Oncology (NCCN Guidelines) have on people with cancer and their families. This special issue of JNCCN_Journal of the National Comprehensive Cancer Network presents these Highlights as a synopsis of the proceedings.

During the conference, esteemed NCCN Guidelines Panel members presented the latest best practices in the treatment of patients with cancer and NCCN Guidelines updates for various diseases, including bladder, breast, colorectal, kidney, lung, and pancreatic cancers, as well as chronic lymphocytic leukemia, gynecologic malignancies, Hodgkin lymphoma, neuroendocrine tumors, non-Hodgkin's lymphomas, soft tissue sarcoma, and the latest NCCN Guideline-myeloproliferative neoplasms.

NCCN experts also discussed frontline supportive care and screening issues. For example, Paul Cinciripini, PhD, of The University of Texas MD Anderson Cancer Center, discussed the benefits of smoking cessation for patients with cancer, as well as the latest advances and best avenues for intervention. Kenneth Offit, MD, MPH, of Memorial Sloan Kettering Cancer Center, discussed personalized medicine. Dr. Offit noted that practitioners could reduce deaths by up to $60 \%$ by paying attention to known risk genetic and lifestyle risk factors for cancer. He also presented the 2017 NCCN Guidelines for Genetic/Familial High-Risk Assessment, which have begun to incorporate data to guide screening based on age-specific risks of genes included on multigene panels.

Stephanie Andrews, MS, ANP-BC, of Moffitt Cancer Center, discussed the management of adverse events for immunotherapy and how to identify them. She noted that enthusiasm for immunotherapy agents should be tempered by a healthy respect for their power. NCCN has devised a teaching and monitoring tool for immunotherapy available at NCCN.org, and is in the process of developing clinical practice guidelines in collaboration with ASCO specifically for the management of immunotherapy-related toxicities in patients with cancer.

The 22nd Annual Conference also featured one of the NCCN's seminal roundtables, opening with a timely expert discussion of disparities in oncology care. Moderated by Clifford Goodman, $\mathrm{PhD}$, from the Lewin Group, the session featured Shauntice Allen, $\mathrm{PhD}$, University of Alabama at Birmingham Comprehensive Cancer Center; Moon S. Chen Jr, PhD, MPH, University of California Davis Comprehensive Cancer Center; Anne Filipic, Enroll America; Edith Mitchell, MD, Sidney Kimmel Cancer Center at Jefferson; and Phyllis Pettit Nassi, MSW, Huntsman Cancer Institute at the University of Utah.

The panelists noted that cancer is the leading cause of death among some communities, and there is an opportunity to make real gains by applying current

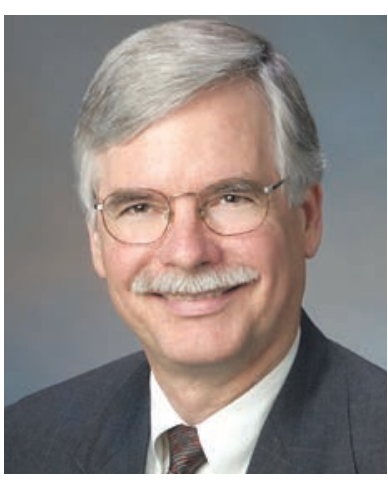

Robert W. Carlson, MD

Robert W. Carlson, MD, joined NCCN as CEO in January 2013 after numerous leadership positions, including Chair of the NCCN Breast Cancer Panel and several NCCN Task Forces. He has also been a member of the NCCN Breast Cancer Risk Reduction Panel, Breast Cancer Disease-Specific Executive Committee, CME Advisory Committee, Guidelines Steering Committee, and Board of Directors. Previously, Dr. Carlson was Professor of Medicine in the Division of Oncology and Stanford Medical Informatics at Stanford University Medical Center. Dr. Carlson received his medical degree from Stanford University Medical School and completed his internship and junior residency in internal medicine at Barnes Hospital Group in St. Louis. He returned to Stanford for his senior residency and postdoctoral fellowship in medical oncology. Dr. Carlson is board certified in internal medicine and holds a subspecialty certification in medical oncology. Dr. Carlson is a member of several medical societies. He lectures extensively and has authored or co-authored over 200 articles, abstracts, and book chapters. 
medical knowledge to these populations. They also spoke of the need to overcome biases to deliver what the individual patient needs, and the need for experts to come out of "silos" and work together. The roundtable ended with panelists challenging attendees to share their opinions with lawmakers so that their voices can be heard.

During Friday's keynote session, which I had the pleasure to moderate, Lucy Kalanithi, MD, widow of the late Paul Kalanithi, MD, author of When Breath Becomes Air, joined Paul's oncologist, Heather Wakelee, MD, of Stanford Cancer Institute, for the first time in public. Together, they recalled the moments with Paul that most influenced their lives and the way they practice medicine.

"Paul faced his cancer in a very, very brave manner," said Dr. Wakelee, who had worked with him at Stanford but didn't get to know him until he was her patient. "What he captures in the book is the essence of what we try to do to help our patients with cancer. Even though he was dying since the time I met him, he really lived."

"Time stood still and sped up at the same time, especially when [Paul] was getting really sick," Dr. Lucy Kalanithi said. "There was this sense of time standing still that came out of the [realization] that the future is not guaranteed."

\section{Additional On-Site Activities}

The conference also gave NCCN the opportunity to launch several exciting new projects. First, JNCCN launched its latest endeavor, JNCCN 360 (JNCCN360.org), a network of tumor-specific microsites that provides users with carefully curated content tailored specifically to the oncology professional audience.

JNCCN 360 includes video discussions with clinical leaders, medical literature summaries, news briefs, and patient resources. One of the most exciting features is the JNCCN Spotlights series, which discusses the safe and effective use of singleagent therapies from within the NCCN Guidelines and the NCCN Drugs \& Biologics Compendium (NCCN Compendium). JNCCN Spotlights provide clinical recommendations from practitioners who have hands-on experience with that therapy, including principal investigators and other thought leaders in oncology. Currently available for breast and non-small cell lung cancers, JNCCN 360 microsites for additional cancer types are expected to launch in 2017.

NCCN also launched the NCCN Radiation Therapy (RT) Compendium (NCCN.org/RTcompendium). RT recommendations contained in the compendium are derived directly from the library of NCCN Guidelines, and the compendium provides guidance on all RT modalities recommended within the NCCN Guidelines, including intensity-modulated RT, intraoperative RT, stereotactic radiosurgery/ stereotactic body RT/stereotactic ablative RT, and others.

Other programs held on site included the 2017 Nursing Program: Advancing Oncology Nursing, the NCCN Fellows Recognition Program: New Horizons in Quality Cancer Care, and various lunch and dinner symposia. Finally, the NCCN General Poster Session featured more than 95 original studies focused on clinical oncology, epidemiology/risks, best practices in implementation and use of clinical practice guidelines, quality improvement, outcomes and health services research, and others.

The NCCN Annual Conference is only one facet of the work that NCCN does as we strive to be the world leader in defining and advancing high-quality, high-value cancer care. We hope you will join us for the NCCN 23rd Annual Conference, March 22 to 24, 2018, at the Rosen Shingle Creek in Orlando, Florida. In the meantime, please visit NCCN.org for more information, to access programs such as the NCCN Guidelines and to watch, and participate in, NCCN's continuing evolution. 